\title{
MODULES AND QUADRATIC FORMS OVER POLYNOMIAL ALGEBRAS
}

\author{
M.-A. KNUS AND M. OJANGUREN
}

\begin{abstract}
Let $D$ be a finite dimensional division algebra over a field $k$. Projective $D[x, y]$-modules of rank $>2$ are free. Projective ideals of $D[x$, $y$ ], $D$ a quaternionic division algebra, which are not free, are used to construct regular quadratic $k[x, y]$-modules which are not extended from $k$.
\end{abstract}

\section{Projective modules.}

Proposition 1.1. Let $D$ be a finite dimensional division algebra over a field $k$. Let $R=k[x, y]$ be the polynomial algebra in two variables and $A=$ $D[x, y]$. Then every finitely generated projective $A$-module of rank $\geqslant 2$ is free.

Proof. Let $S=k[x], C=D[x]$ and for each maximal ideal $m$ of $S$, $C_{m}=S_{m} \otimes_{S} C$. Let $P$ be a finitely generated projective $A$-module with $n=\operatorname{rank} P \geqslant 2$. Since $K_{0}(A)=K_{0}(D), P$ is stably free. The maximal spectrum of $S_{m}[y]$ is the union of two 1-dimensional sets [2, III, Proposition 3.13], hence by the cancellation theorem of Bass and Schanuel [2, IV, Theorem 3.1], $C_{m} \otimes_{C} P \cong C_{m}[y]^{n}$. In other words, the $C[y]$-module $P$ is locally extended from $C$. By a slight generalisation of Quillen's theorem [10, Theorem 1] $P$ is extended from $C$, i.e. $P \cong C[y] \otimes_{C} Q$ for some $C$-module $Q$. Since projective $C$-modules are free, so is $P$.

REMARK 1.2. Examples of nonfree projective ideals in $D[x, y]$ are given in [8].

2. Quadratic modules. Let $R$ be a commutative ring, $V$ a finitely generated $R$-module and $f: V \times V \rightarrow R$ a bilinear symmetric pairing. We call the pair $(V, f)$ a regular quadratic $R$-module if the map of $V$ into $\operatorname{Hom}_{R}(V, R)$ induced by $f$ is an isomorphism. If $(V, f)$ is a regular quadratic $R$-module and $S$ is any commutative $R$-algebra, then $\left(S \otimes_{R} V, 1_{S} \otimes f\right)$ is a regular quadratic $S$-module. A regular quadratic $S$-module is extended from $R$ if it is isomorphic to some $\left(S \otimes_{R} V, 1_{S} \otimes f\right)$. Let $R=k\left[t_{1}, \ldots, t_{n}\right]$ be a polynomial algebra in $n$ variables over a field $k$ of characteristic not 2 . For $n=1$, Harder proved that regular quadratic $R$-modules are always extended from $k$ (see [5, Theorem 13.4.1]). In [9] S. Parimala proved that regular quadratic $R$-modules of rank 2 are always extended, and constructed

Received by the editors November 24, 1976.

AMS (MOS) subject classifications (1970). Primary $13 \mathrm{C} 10$.

๑ American Mathematical Society 1977 
infinitely many rank 4 quadratic modules over $\mathbf{R}[x, y]$ which are not extended. Very recently $M$. S. Raghunathan proved that regular quadratic $R$-modules are extended if the $\operatorname{Brauer}$ group $\operatorname{Br}(k)$ of $k$ has no element of order two [11]. Here we show that this result is wrong in general without the condition on $\operatorname{Br}(k)$.

Proposition 2.1. Let $k$ be a field of characteristic not 2 or 3 over which there exists a quaternionic division algebra $D$. There exist two regular quadratic $k[x, y]$-modules of rank 3 (resp. 4) which are not extended from $k$.

Proof. We first construct a central separable $k[x, y]$-algebra which is not extended from $k$. Put $R=k[x, y]$ and $A=D[x, y]$. According to [8], $A$ contains a nonfree projective left ideal $I$ and the central separable $R$-algebra $B=\operatorname{End}_{A}(I)$ is not isomorphic to $A$. Clearly $A /(x, y) A \cong B /(x, y) B \cong D$ as $k$-algebras, hence if $B$ were extended, then it would be isomorphic to $A$. We now define quadratic forms on $A$ and $B$. The reduced trace and the reduced norm of $D$ induce, by scalar extension, the reduced trace $T_{A}$ and the reduced norm $N_{A}$ of $A$. By general results on central separable algebras [3], [6], $B$ also has a reduced trace $T_{B}$ and a reduced norm $N_{B}$. Since $A$ and $B$ are free of rank four over $R, N_{A}$ and $N_{B}$ define, in the usual way, quadratic structures on $A$ and $B$. The quadratic modules $A$ and $B$ are easily seen to be regular (for instance by tensoring over $k$ with the algebraic closure of $k$ and using faithfully flat descent). Now notice that, if $B$ is extended from $k$, then $B$ must be isometric to $A$ (go modulo $(x, y)$ and use the fact that the reduced norm "commutes" with scalar extension). Following N. Jacobson $[4$, p. 185] and using a result of Ancochea [1], we show that $A \cong B$ as quadratic modules implies an isomorphism of algebras, contradicting the result obtained above. Let ': $A \rightarrow B$ be an isomorphism of $R$-modules such that $N_{B}\left(a^{\prime}\right)=N_{A}(a)$ for every element $a$ in $A$. In particular, $1=N_{A}(1)=N_{B}\left(1^{\prime}\right)$. Hence, $1^{\prime}$ is a unit of $B$, and replacing $a \mapsto a^{\prime}$ by $a \mapsto a^{\prime}\left(1^{\prime}\right)^{-1}$, we can assume that $1^{\prime}=1$. By linearity $r^{\prime}=r$ for every $r$ in $R$. Then, for any $a$ in $A$,

$$
\begin{aligned}
N_{B}\left((r+a)^{\prime}\right) & =N_{B}\left(r^{\prime}+a^{\prime}\right)=N_{B}\left(r+a^{\prime}\right) \\
& =r^{2}+r T_{B}\left(a^{\prime}\right)+N_{B}\left(a^{\prime}\right), \\
N_{A}(r+a) & =r^{2}+r T_{A}(a)+N_{A}(a) .
\end{aligned}
$$

Since $R$ is infinite this implies that $T_{A}(a)=T_{B}\left(a^{\prime}\right)$. From this, we first deduce that ' is a semi-isomorphism in the sense of [1], i.e. $(a b+b a)^{\prime}=a^{\prime} b^{\prime}+b^{\prime} a^{\prime}$. Then, using the fact that $A \otimes_{R} k(x, y) \cong B \otimes_{R} k(x, y)$, one can conclude, as in [1], that ' is an isomorphism or an anti-isomorphism. Since $A$ is isomorphic to its opposite, $A$ and $B$ are isomorphic. Since $(s, t) \mapsto T_{B}(s t)$ is a regular pairing, in order to show that $(a b+b a)^{\prime}=a^{\prime} b^{\prime}+b^{\prime} a^{\prime}$, it suffices to show that, for every $c$ in $A, T_{B}\left((a b+b a)^{\prime} c^{\prime}\right)=T_{B}\left(\left(a^{\prime} b^{\prime}+b^{\prime} a^{\prime}\right) c^{\prime}\right)$. We first claim that, for every $a$ in $A$,

$$
T_{A}\left(a^{2}\right)=T_{B}\left(\left(a^{\prime}\right)^{2}\right)
$$




$$
T_{A}\left(a^{3}\right)=T_{B}\left(\left(a^{\prime}\right)^{3}\right)
$$

In fact $a$ and $a^{\prime}$ satisfy the Cayley-Hamilton polynomials [3], [6]

$$
\begin{gathered}
a^{2}-a T_{A}(a)+N_{A}(a)=0, \\
\left(a^{\prime}\right)^{2}-a^{\prime} T_{B}\left(a^{\prime}\right)+N_{B}\left(a^{\prime}\right)=0 .
\end{gathered}
$$

Taking traces of (3) and (4) we get (1). Multiplying (3) by $a$, (4) by $a^{\prime}$ and using (1) we get (2).

Now, since $T_{B}(s t)=T_{B}(t s)$, it is easily checked that

$$
2 T_{B}\left((a b)^{\prime} c^{\prime}\right)=T_{B}\left(\left((a b+c)^{\prime}\right)^{2}-\left((a b)^{\prime}\right)^{2}-\left(c^{\prime}\right)^{2}\right) .
$$

Using (1) we then get $T_{B}\left((a b)^{\prime} c^{\prime}\right)=T_{A}(a b c)$. Similarly, from

$$
\begin{aligned}
3 T_{B}\left(\left(a^{\prime} b^{\prime}+\right.\right. & \left.\left.b^{\prime} a^{\prime}\right) c^{\prime}\right) \\
= & T_{B}\left(\left((a+b+c)^{\prime}\right)^{3}-\left((a+b)^{\prime}\right)^{3}-\left((a+c)^{\prime}\right)^{3}\right. \\
& \left.-\left((b+c)^{\prime}\right)^{3}+\left(a^{\prime}\right)^{3}+\left(b^{\prime}\right)^{3}+\left(c^{\prime}\right)^{3}\right)
\end{aligned}
$$

and from (2) we get $T_{B}\left(\left(a^{\prime} b^{\prime}+b^{\prime} a^{\prime}\right) c^{\prime}\right)=T_{A}((a b+b a) c)$. This shows that ' is a semi-isomorphism of $R$-algebras.

REMARK 2.1. Since $N_{B}(1)=1$, the submodule $R$ of $B$ has an orthogonal complement $B_{0}$ of rank 3 . Since $B$ is not extended from $k$, neither is $B_{0}$. This is in contrast with the result of S. Parimala [9] asserting that quadratic modules of rank 2 are always extended from $k$. Notice that $B_{0}$ must be indecomposable.

REMARK 2.2. The Clifford algebras $C_{A}$ and $C_{B}$ of the quadratic modules $A$ and $B$ are both isomorphic to $M_{2}(A)$. This can be shown in the following way. Since $A$ and $B$ are stably isometric, $C_{A}$ and $C_{B}$ (which are both central separable $R$-algebras) are Brauer equivalent over $R$. Hence $M_{n}\left(C_{A}\right) \cong$ $M_{n}\left(C_{B}\right)$ for some $n$. According to [8], to prove $C_{A} \cong C_{B}$, it suffices to show that, for any left $C_{A}$-module $I, I^{n} \cong C_{A}^{n}$ implies that $I$ is a bimodule. We prove that any such $I$ is in fact free. Observe that $C_{A} \cong M_{2}(A)[7$, p. 116, Corollary 3.3]. Hence by Morita theory, we only have to show that $A^{2} \otimes_{C_{A}} I$ is free as left $A$-module. This is a special case of Proposition 1.1.

We thank A. Geramita for suggesting the following proposition, which shows that there exist nonextended quadratic $\mathbf{R}[x, y]$-modules of arbitrarily large rank.

Proposition 2.3. Let $B_{0}$ be the quadratic $\mathbf{R}[x, y]$-module of Remark 2.1 (with $k=\mathbf{R}$ ). Let $M$ be the orthogonal direct sum of $n$ copies of $B_{0}$. Then $M$ is not extended from $\mathbf{R}$.

Proof. Let $f\left(X_{1}, X_{2}, X_{3}\right)$ be the quadratic form with coefficients in $\mathbf{R}[x, y]$ corresponding to $B_{0}$. Observe that for any real maximal ideal $m=(x-\xi$, $y-\eta)$ of $\mathbf{R}[x, y]$ the $\mathbf{R}$-algebra $B / m B$ is the usual quaternion algebra. Hence replacing $x, y$ by $\xi, \eta$ in the coefficients of $f\left(X_{1}, X_{2}, X_{3}\right)$ yields a positive definite quadratic form over $\mathbf{R}$. The quadratic form corresponding to $M$ is 
$f\left(X_{11}, X_{12}, X_{13}\right)+\cdots+f\left(X_{n 1}, X_{n 2}, X_{n 3}\right)$, and it suffices to show that it does not represent 1 over $\mathbf{R}[x, y]$. Suppose that, for some $U_{i j} \in \mathbf{R}[x, y]$,

$$
F(x, y)=f\left(U_{11}, U_{12}, U_{13}\right)+\cdots+f\left(U_{n 1}, U_{n 2}, U_{n 3}\right)=1 \text {. }
$$

Then, for any $\xi, \eta \in \mathbf{R}, F(\xi, \eta)=1$ and each $f\left(U_{i 1}(\xi, \eta), U_{i 2}(\xi, \eta), U_{i 3}(\xi, \eta)\right)$, being nonnegative, must lie between 0 and 1 . Hence all the $f\left(U_{i 1}(x, y), U_{i 2}(x\right.$, $\left.y), U_{i 3}(x, y)\right)$ are constant and at least one of them is $\neq 0$. This is impossible because $B_{0}$ is indecomposable and therefore $f\left(X_{1}, X_{2}, X_{3}\right)$ does not represent a nonzero constant over $\mathbf{R}[x, y]$.

ADDED IN PROOF. The argument in 2.3 can be refined to give examples of nonextended quadratic modules of any rank $\geqslant 3$.

\section{REFERENCES}

1. G. Ancochea, On semi-automorphisms of division algebras, Ann. of Math. 48 (1947), 147-153.

2. H. Bass, Algebraic K-theory, Benjamin, New York, 1968.

3. A. Grothendieck, Le groupe de Brauer. I, Dix exposés sur la cohomologie des schémas, North-Holland, Amsterdam, 1968.

4. N. Jacobson, Some groups of transformations defined by Jordan algebras. I, J. Reine Angew. Math. 201 (1959), 178-195.

5. M. Knebusch, Grothendieck-und Wittringe von nichtausgearteten symmetrischen Bilinearformen, Sitzungsber. Heidel. Akad. Wiss. Math. Natur. Kl. (1969/70), 89-157.

6. M.-A. Knus and M. Ojanguren, Théorie de la descente et algèbres d'Azumaya, Lecture Notes in Math. vol. 389, Springer, Berlin and New York, 1974.

7. T. Y. Lam, Algebraic theory of quadratic forms, Benjamin, New York, 1973.

8. M. Ojanguren and R. Sridharan, Cancellation of Azumaya algebras, J. Algebra 18 (1971), 501-505.

9. S. Parimala, Failure of a quadratic analogue of Serre's conjecture, Bull. Amer. Math. Soc. 82 (1976), 962-964.

10. D. Quillen, Projective modules over polynomial algebras, Invent. Math. 36 (1976), 167-171.

11. M. S. Raghunathan, Principal bundles on affine space, Bombay, 1976 (preprint).

Mathematisches Seminar, Eidgenössische Technische Hochschule, Zürich, SwitzerLAND (Current address of M.-A. Knus)

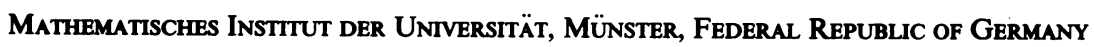

Current address (M. Ojanguren): Institut de Mathématiques, Université de Lausanne, Lausanne, Switzerland 